EPJ Web of Conferences 110,01023 (2016)

DOI: 10.1051/epjconf/201611001023

(C) Owned by the authors, published by EDP Sciences, 2016

\title{
INITIATION OF IGNITION OF HIGHLY FUEL PARTICLES IN THE FLOW OF HIGH TEMPERATURE MEDIUM
}

\author{
S.V. Syrodoy ${ }^{1, \text { a }}$, N.Y. Gutareva ${ }^{1}$, V.V. Salomatov ${ }^{2}$ \\ ${ }^{1}$ National Research Tomsk Polytechnic University, 634050 Tomsk, Russia
}

${ }^{2}$ S.S. Kutateladze Institute of Thermophysics, Siberian Division of the Russian Academy of Sciences, 634040 Novosibirsk, Russia

\begin{abstract}
An approximate analytical solution of ignition of the fuel porous particles has been brought. The impact of thermal radiation on the characteristics and conditions of the ignition has been considered. The verification of the solution has been obtained by comparing the calculated characteristics (delay time of ignition and the ignition temperature) with similar values obtained using the previously derived formulas.
\end{abstract}

\section{Introduction}

According to experts [1] coal will continue to be the main fuel and energy resource. However, high anthropogenic (large emissions of nitrogen oxides and sulfur) traditional ways of organizing the combustion, creates prerequisites for the development of new innovative methods of burning coal. The latter can be based on improving methods of fuel preparation (coal ultrafine grinding [2]) and burning (the use of plasma [3] or microwave illumination [4]). However, these methods entail an increase of costs of reconstruction and operation of boiler equipment. One of the most promising technical solutions is the combustion of coal in coalwater fuel (CWF).

Coal-water fuel - mixture of fine-grained coal (50-60\%) and water (49-39\%) with the addition of additives and plasticizers $(1 \%)$. In general case the drop of CWF is highly porous structure with water-filled pores. It should be noted that this technology (despite more than fifty years of history of development [5]) has not been widely used in thermal power plants. The last is, most likely, due to inadequate basic aspects conceived of the theory of ignition of such fuels. Numerical methods [6-8] allow to make calculations (even with full flowing together of the complex physic-chemical processes) thermal preparation and ignition of particles of CWF. However, such techniques are difficult to apply when designing combustion equipment of boiler units. The latter is due to high demands on high-performance computer technology and the availability of special programmatic components. For this reason, the analytical solution of the problem of ignition of fuel is the most appropriate for the development of the normative recommendations of designing combustion equipment the water-coal boiler units.

The paper discusses approximate analytical solution of the ignition CWF particles within a model of highly porous structure with low thermal characteristics.

\section{Mathematical formulation of the problem the ignition}

In the initial time $(t=0)$ a drop of water coal fuel, which represents a highly porous carbon structure with water-filled pores, gets in a high temperature environment. Obviously, such high water-cut fuel particle will not ignite until its complete "dehydration". Porous structure with low thermal conductivity and heat capacity remains after complete drying. A further thermal effect on the particle initiates inflammation. Solving problems of inert heating [9] and evaporation [10] have already been known.

\footnotetext{
${ }^{a}$ Corresponding author: ssyrodoy@yandex.ru
}

This is an Open Access article distributed under the terms of the Creative Commons Attribution License 4.0, which permits unrestricted use, distribution, and reproduction in any medium, provided the original work is properly cited. 


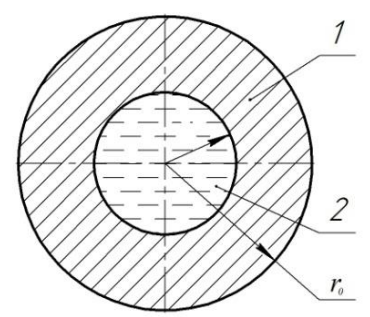

Figure 1. The scheme of the problem of ignition. 1-Dehydrated part of the fuel; 2-the original CWF.

Mathematical formulation of the problem of ignition of porous carbon particles consists of the following system of non-stationary differential equations in private derivatives:

The equation of heat conduction with a chemical heat formation:

$$
\frac{1}{a} \frac{\partial T_{1}(r, t)}{\partial t}=r^{-2} \frac{\partial}{\partial r}\left[r^{2} \frac{\partial T_{1}(r, \tau)}{\partial r}\right]+\frac{Q z}{c} \cdot \exp \left[-\frac{E}{R \cdot T_{1}(r, t)}\right]
$$

The system of equations (1-3) has been solved under the following boundary conditions and closing relations

$$
\begin{gathered}
0<t<t_{\text {ign }} \quad 0<r<r_{0} \quad T(r, 0)=T_{0} \\
-\lambda \frac{\partial T\left(r_{0}, t\right)}{\partial r}=\alpha \cdot\left(T_{E}-T\left(r_{0}, \tau\right)\right)+\sigma \cdot\left(T_{E}^{4}-T\left(r_{0}, \tau\right)^{4}\right) \\
-\lambda \frac{\partial T(0, t)}{\partial r}=0
\end{gathered}
$$

Taking into consideration that the porous frame has a high thermal resistance, it is possible to tell with confidence that ignition will take place in a thin surface layer. In this case, the system (1-3) can lead to the following form:

$$
\begin{gathered}
\frac{\partial T_{1}(x, t)}{\partial t}=a \cdot\left[\frac{\partial^{2} T_{1}(x, \tau)}{\partial x^{2}}\right]+\frac{Q z}{c} \cdot \exp \left[-\frac{E}{R \cdot T_{1}(x, t)}\right] \\
-\lambda \frac{\partial T(0, t)}{\partial x}=\alpha_{c}\left(T_{C}-T(0, \tau)\right)+\sigma_{c} \cdot\left(T_{E}^{4}-T(0, \tau)^{4}\right) \\
-\lambda \frac{\partial T(\infty, t)}{\partial x}=0
\end{gathered}
$$

The system (4-6) has no analytical solution, due to nonlinear boundary conditions [radiant component of heat transfer is in equation (5)]. To avoid this nonlinearity, you can resort to betake of linearization type [11]:

under condition

under condition

$$
\sigma \cdot\left(T_{E}^{4}-T\left(r_{0}, t\right)^{4}\right) \approx \sigma \cdot T_{E}^{4}
$$

$$
\frac{T\left(r_{0}, t\right)}{T_{E}} \leq 0.65
$$

$$
\frac{T\left(r_{0}, t\right)}{T_{E}}>0.65
$$

$$
\sigma \cdot\left(T_{E}^{4}-T\left(r_{0}, t\right)^{4}\right) \approx 4 \cdot \sigma \cdot T_{E}^{3} \cdot\left(T_{E}-T\left(r_{0}, t\right)\right)
$$


Let's consider dimensionless variables, using the transformation of Frank-Kamenetskii [12]:

$$
\begin{aligned}
& \theta=\frac{E}{R \cdot T_{*}^{2}} \cdot\left(T-T_{*}\right) \quad \theta_{E}=\frac{E}{R \cdot T_{*}^{2}} \cdot\left(T_{E}-T_{*}\right) \quad \theta_{0}=\frac{E}{R \cdot T_{*}^{2}} \cdot\left(T_{0}-T_{*}\right) \quad \xi=\frac{x}{x_{a}} \quad \beta=\frac{R \cdot T_{*}}{E} \\
& \tau=\frac{t}{t_{a}} \quad x_{a}=\sqrt{a \cdot t_{a}} \quad B i=\frac{\alpha \cdot x_{a}}{\lambda} \quad K i=\frac{E}{R \cdot T_{*}^{2}} \cdot \frac{x_{a}}{\lambda} \cdot \sigma_{c} \cdot T_{E}^{4} \\
& t_{a}=\frac{R \cdot T_{*}^{2}}{E} \cdot \frac{c}{Q z} \exp \left(\frac{E}{R \cdot T^{*}}\right)
\end{aligned}
$$

As a result, the system (4)-(6) is converted to the following form:

$$
\begin{aligned}
& \frac{\partial \theta(\zeta, K)}{\partial \tau}=\frac{\partial^{2} \theta(\zeta, K)}{\partial \zeta^{2}}+\exp \left[\frac{\theta}{1+\beta \cdot \theta}\right] \\
& \theta(\xi, 0)=\theta_{0} \\
& -\frac{\partial \theta(0, \tau)}{\partial \xi}=B i \cdot\left(\theta_{E}-\theta(0, \tau)\right)+K i
\end{aligned}
$$

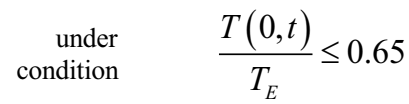

$$
\begin{aligned}
& -\frac{\partial \theta(0, \tau)}{\partial \xi}=B i_{\Sigma}\left(\theta_{E}-\theta(0, \tau)\right) \\
& \begin{aligned}
\text { under } & \frac{T(0, t)}{T_{E}}>0.65
\end{aligned} \\
& -\frac{\partial \theta(\infty, \tau)}{\partial \xi}=0
\end{aligned}
$$

The solution of the problem of ignition will be carried out in the framework of the adiabatic theory [13]. Accordingly, the ignition process can be divided into two consequent stages (Figure.2.):

1. Inert heating to a temperature (on the surface of particles) start of ignition;

2. Ignition of the fuel.

The expression (9) can be divided into two equations based on the above assumptions:

$$
\begin{gathered}
\frac{\partial \theta(r, \tau)}{\partial \tau}=\frac{\partial^{2} \theta}{\partial \zeta^{2}} \\
\frac{\partial \theta(r, \tau)}{\partial \tau}=\exp \left[\frac{\theta}{1+\beta \cdot \theta}\right]
\end{gathered}
$$

The solution of equation (14) has been discussed in detail in [14]. We'll carry out the method of operational calculus [15] the solution of the equation (13), most frequently in engineering practice, the ratio of the temperatures

$\left((T(0, t)) / T \_c\right) \leq 0.65$. Accordingly, we'll consider the following system of equations:

$$
\begin{gathered}
\frac{\partial \theta(r, \tau)}{\partial \tau}=\frac{\partial^{2} \theta}{\partial \zeta^{2}} \\
\theta(\xi, 0)=\theta_{0}
\end{gathered}
$$




$$
\begin{gathered}
-\frac{\partial \theta(0, \tau)}{\partial \xi}=K i(\tau) \\
-\frac{\partial \theta(\infty, \tau)}{\partial \xi}=0
\end{gathered}
$$

where:

$$
K i(\tau)=B i \cdot\left(\theta_{E}-\theta(0, \tau)\right)+K i
$$

The ignition process is fast, according to [15], the display $f(\tau) \rightarrow F(s)$ is found from the condition that $\tau-$ "little" and the time display s- great. As a result, the system (15)-(18) has been brought to the form:

$$
\begin{gathered}
S \theta(\xi, S)-\theta_{0}=\frac{\partial \theta^{2}(\xi, S)}{\partial \xi} \\
\theta_{L}(\xi, 0)=\frac{\theta_{0}}{S} \\
-\frac{\partial \theta_{L}(0, S)}{\partial \xi}=K i(S) \\
\frac{\partial \theta_{L}(\infty, S)}{\partial \xi}=0
\end{gathered}
$$

To solve the system (19)-(22) we need to carry the proper change of variables:

$v(\xi, S)=\theta_{L}(\xi, S)-\frac{\theta_{0}}{S}$

Accordingly, the expression (19) is converted to the form:

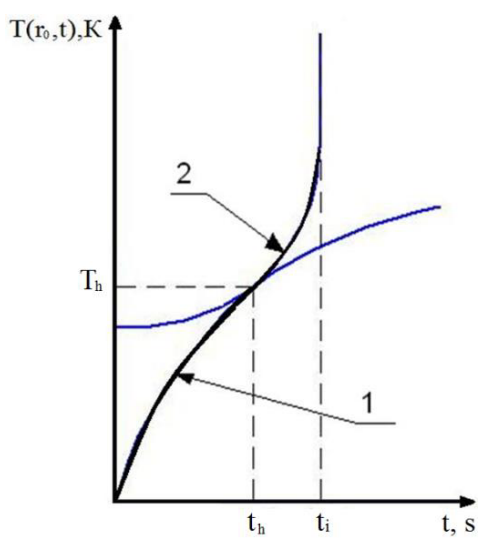

Figure 2. Changes in the surface temperature of the fuel particles in the ignition.1st Stage of warming; 2 inflammation.

$$
v^{\prime \prime}(\xi, S)=S \cdot v(\xi, S)
$$

The solution of this equation will be the following relationship:

$$
v(\xi, S)=A \cdot e^{-\sqrt{S} \cdot \xi}+B \cdot e^{\sqrt{S} \cdot \xi}
$$

You might guess that the constant B will be equal to zero, respectively equation (25) will take the following form: 


$$
v(\xi, S)=A \cdot e^{-\sqrt{S} \cdot \xi}
$$

We'll need to satisfy (26) boundary condition (21):

$$
v(\xi, S)=A \cdot e^{-\sqrt{S} \cdot \xi=0} \cdot \sqrt{S} \equiv K i(S)
$$

Therefore, the constant of integration is:

$$
A=\frac{K i(S)}{\sqrt{S}}
$$

Substituting (28) into equation (25) we'll receive:

$$
v(\xi, S)=K i(S) \cdot \frac{e^{-\sqrt{S} \cdot \xi}}{S}
$$

Let's move from the field of displays back to the originals:

$$
v(\xi, \tau)=L^{-1}\left(K i(S) \cdot F_{L}(S)\right)
$$

where:

$$
F(S)=\frac{e^{-\sqrt{S} \cdot \xi}}{S}
$$

As it has already been known [15] at the transition to the artwork of the originals is necessary to consider the convolution property mappings, respectively:

$$
v(\xi, \tau)=\int_{0}^{\tau} K i(\eta) \cdot F(\tau-\eta) d \eta=\int_{0}^{\tau} K i(\eta) \cdot \frac{1}{\sqrt{\pi(\tau-\eta)}} \cdot e^{-\frac{\xi^{2}}{4(\tau-\eta)}} d \eta
$$

Taking into consideration that:

$$
v(\xi, S)=\theta_{L}(\xi, S)-\frac{\theta_{H}}{S}
$$

the expression (32) can be reduced to the following form:

$$
\theta(\xi, \tau)-\theta_{0}=\int_{0}^{\tau} K i(\eta) \cdot \frac{1}{\sqrt{\pi(\tau-\eta)}} \cdot e^{-\frac{\xi^{2}}{4(\tau-\eta)}} d \eta
$$

In order to get rid of the complex of

$1 / \sqrt{\pi(\tau-\eta)}$ we'll decompose the function $\operatorname{Ki}(\eta)$ in a Taylor series:

$$
\begin{aligned}
K i(\eta) & =K i(\tau)+K i(\tau) \cdot(\eta-\tau)+O\left(\tau^{2}\right) \\
\eta & \approx \tau
\end{aligned}
$$

Accordingly, the equation (34) can be represented in the following way:

$$
\theta(\xi, \tau)-\theta_{0}=\int_{0}^{\tau}\left[K i(\eta)+K i^{\prime}(\tau)(\eta-\tau)+O\left(\tau^{2}\right)\right] \times \frac{1}{\sqrt{\pi(\tau-\eta)}} \cdot e^{-\frac{\xi^{2}}{4(\tau-\eta)}} d \eta
$$

It is obvious that the following expression is the solution of the equation (36): 


$$
\theta(\xi, \tau)=\theta_{0}+\left\{B i \cdot\left[\theta_{E}-\theta_{(0, \tau)}\right]+K i\right\} \times \frac{2}{\sqrt{\pi}}\left(\sqrt{\tau} \cdot e^{-\frac{\xi^{2}}{4 \tau}}-\frac{\xi}{2} \sqrt{\pi}\left[\operatorname{erfc}\left(\frac{\xi}{2 \cdot \sqrt{\tau}}\right)\right]\right)
$$

where "erfc" is the error function of Gauss [16]. The ignition process is localized in the most energy-intensive location (on the surface) particles, therefore:

$$
\theta(0, \tau)=\theta_{0}+\left\{B i \cdot\left[\theta_{E}-\theta_{(0, \tau)}\right]+K i\right\} \cdot \frac{2 \sqrt{\tau}}{\sqrt{\pi}}
$$

We'll transform (38) relatively $\theta(0, \mathrm{t})$ :

$$
\theta(0, \tau)=\frac{\theta_{0}+\frac{2 \sqrt{\tau}}{\sqrt{\pi}}\left(B i \cdot \theta_{E}+K i\right)}{1+B i \frac{2 \sqrt{\pi}}{\sqrt{\tau}}}
$$

If the magnitude of the temperature we'll choose the temperature of the beginning of ignition, at the boundary of solutions of the equations (13)-(14) executes the condition of cross linking. It is not hard to guess that in this case, the warm-up time (the period from the beginning of exposure to heat until ignition) can be calculated from the following expression:

$$
\tau_{h}=\frac{\pi \cdot \theta_{0}^{2}}{4\left(B i \cdot \theta_{E}+K i\right)^{2}}
$$

It is known [14] that the full induction period can be represented as the sum of times of warming up and ignition of particles:

$$
\tau_{i g n}=\tau_{h}+\tau_{\text {ind }}
$$

where $\tau_{\text {ind }}$ - induction time[14]:

$$
\tau_{\text {ind }}=1+2 \cdot \beta
$$

To determine the minimum (for ignition) temperature of heating of the particles with respect (39) on time and satisfy the condition of "sewing". As a result, after simple transformations we'll get the expression for calculating the temperature of ignition:

$$
\frac{\partial \theta(0, \tau)}{\partial \tau}=\frac{\frac{\sqrt{\tau^{3}}}{\sqrt{\pi}} \cdot\left[Q-B i \cdot \theta_{0}\right]}{\left(1+2 \cdot B i \cdot \sqrt{\frac{\tau}{\pi}}\right)^{2}}
$$

where $\mathrm{Q}=\left(\mathrm{Bi} \cdot \theta_{\mathrm{c}}+\mathrm{Ki}\right)$

Let's transform (43) in dimensional form:

$$
\rho \cdot Q z \cdot \exp \left[-\frac{E}{R \cdot T_{h}}\right] \cdot\left[\frac{\lambda \cdot\left(T_{h}-T_{0}\right)}{\alpha \cdot\left(T_{E}-T_{h}\right)+\sigma \cdot T_{E}^{4}}\right] \cdot \frac{\pi}{2} \cdot\left(1+\frac{\left(T_{h}-T_{0}\right)}{\alpha \cdot\left(T_{E}-T_{h}\right)+\sigma \cdot T_{E}^{4}}\right)^{2}=\alpha \cdot\left(T_{E}-T_{0}\right)+\sigma \cdot T_{E}^{4}
$$

\section{Results and Discussion}

We'll estimate the time and temperature of heating of the fuel particles at variation of heat transfer conditions. We'll carry tested expressions (40) and (44) on the example of calculation of process of ignition of the particles of the N-gunpowder, by comparison with the previously described formulas [13].

There are the dependences of temperatures of heating of fuel particles from the ambient temperature under different conditions of heat exchange, in the case of a convective mechanism in Figure 3. Dependency analysis has shown that in conditions of convective heat transfer a satisfactory consistency (almost in the whole range $\alpha \approx 100 \div 500 \mathrm{~W} /(\mathrm{m} \cdot \mathrm{K})$ ) temperature of heating has been calculated 


\section{Thermophysical Basis of Energy Technologies 2015}

from expression (44) and by the method of [13]. There are the dependences of temperatures of heating of fuel particles from the ambient temperature in the case of convective-radiant heat exchange in Figure 4.

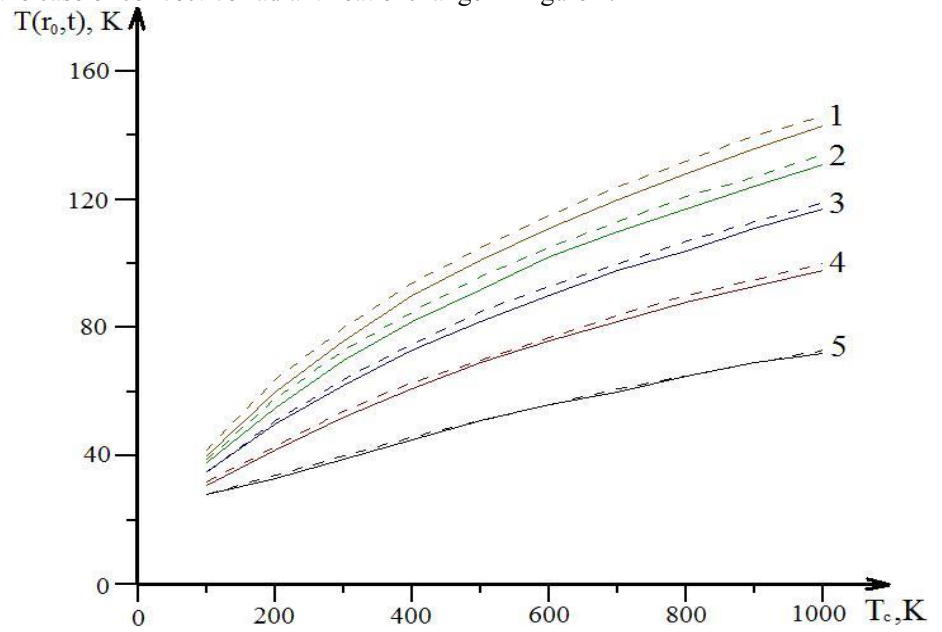

Figure 3. The dependence of the heating temperature (before ignition) of the fuel particles from ambient temperature at different intensities of heat transfer (in the case of only convection). $1-\alpha=500 ; 2-400 ; 3-300 ; 4-200 ; 5-100 \mathrm{~W} /((\mathrm{m} \cdot \mathrm{K}))$ - the temperature of heating has been calculated from expression (44) - the temperature of the heating has been calculated by the formulas [13].

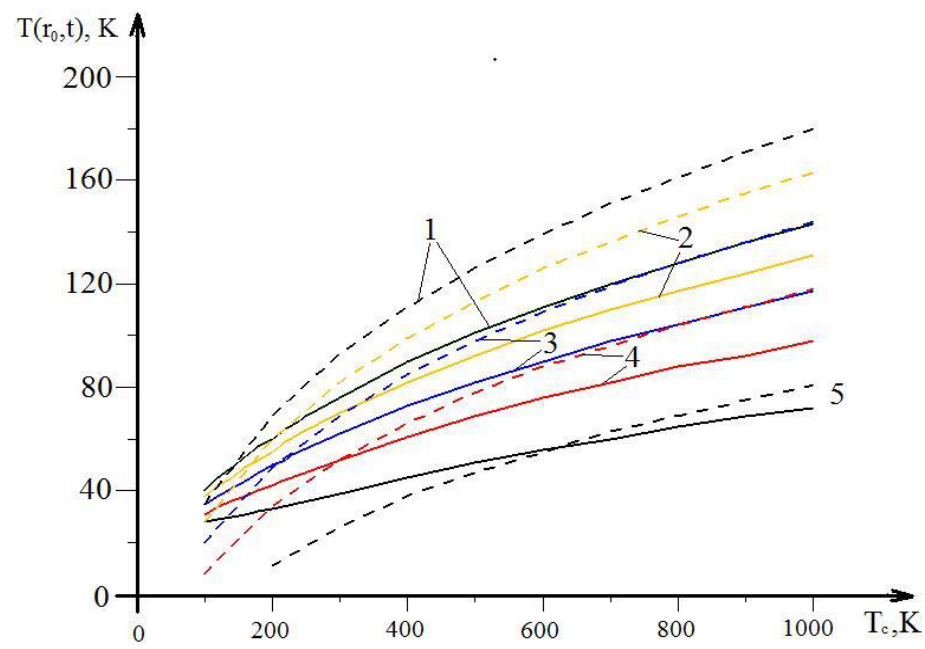

Figure 4. The dependence of the heating temperature of the fuel particles from ambient temperature at different intensities of heat transfer (in the case of convective-radiant heat exchange). $1-\alpha=500 ; 2-400 ; 3-300 ; 4-200 ; 5-100 \mathrm{~W} /((\mathrm{m} \cdot \mathrm{K}))-$ the temperature of heating has been calculated from expression (44) - the temperature of the heating has been calculated by the formulas [13].

Having analyzed the curves, we can say that in the temperature range from $\mathrm{Te}=500 \div 800 \mathrm{~K}$ satisfactory coherence times calculated by the formula (44) given in [13] dependence. The latter may be due to the fact that in [13] considers "quasi-stationary" model of ignition.

Figure 5 shows the dependence of the delay times of the ignition from the heat transfer. Similar to figures 5 and 6 it is safe to say that in case of only convective mechanism of heat exchange there is a satisfactory consistency of the results of calculation by formula (40) and the dependence is given in [13]. As we have already emphasized previously, the latter may be due to the fact that in [13] the solution to the problem of ignition in a "quasi-stationary" approximation. This simplification facilitates greatly the modelling procedure, but may lead to large errors. 


\section{EPJ Web of Conferences}

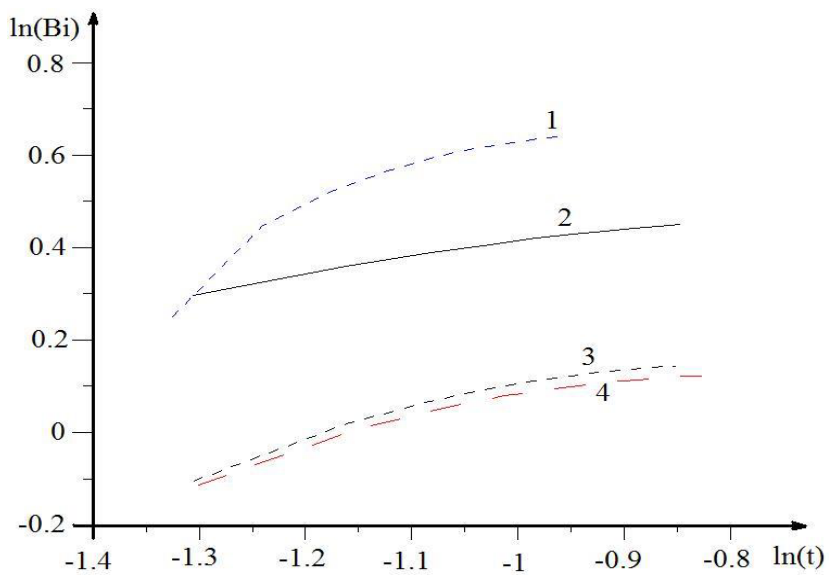

Figure 5.The dependence of the ignition delay of the heat transfer rate, 1,2 - delay time of ignition in the case of convective-radiative heat exchange, 3, 4- the delay time of ignition in the case of convective heat transfer, 1,3 - ignition delay time calculated by the formula [13], 2, 4ignition delay time calculated by the formula (40)

\section{Conclusion}

Analytical solution of the problem of ignition of porous "dehydrated" - water particles has been obtained by simulation results. This formulas allows you quickly and cost-effectively (without the use of energy-intensive, high-performance computer technology) to perform calculations the most important characteristics of ignition (e.g., ignition delay) and to determine the ignition conditions (minimum required temperature of heating). Testing of deduced formulas (40) and (44) has been carried out by comparing the calculation results of ignition delay time and temperature of heating with the already known [13] values. The curve analysis has showed high consistency of the decisions in the case of convective mechanism of heat transfer Such coherence is observed in a narrow $(\approx 600 \div 800 \mathrm{~K})$ range of ambient temperatures in the radiation-convective heating. The latter may be due to different methods of output calculation formulas.

The research has been carried out at the expense of the grant of the Russian Science Foundation (project № 15-19-10003).

\section{References}

1. J.P. Longwell, E.S. Rubin, J. Wilson, Prog. Energy Combust. Sci., 21 (1995)

2. S.V. Alekseenko, A.P. Burdukov, The use of mechanically activated coal in energy technologies (Research and Development, SB RAS in energy efficient technologies. Novosibirsk: Publishing House of SB RAS, 2009)

3. A. Starikovskiy, N. Aleksandrov, Prog. Energy Combust. Sci., 39, 1 (2013)

4. Y.A. Chumakov, A.G.Knyazev, Combustion and explosions, 2, (2012)

5. V.F. Scrubs, A.D. Kokurin, N.I. Fissenko, Production process gases from aqueous suspensions of fuel (Works in the field of chemical technology of fuel. L.: Goskhimizdat, 1959)

6. V.V. Salomatov, S.V. Syrodoy, N.Y. Gutareva, IOP Conf. Series: Materials Science and Engineering, 66, 012040 (2014)

7. V.V. Salomatov, S.V. Syrodoy, N.Y. Gutareva, EPJ Web of Conferences, 82, 01037 (2015)

8. G.V. Kuznetsov, V.V. Salomatov, S.V. Syrodoy, Combustion, Explosion and Shock waves, 51, 4 (2015)

9. V.V. Salomatov, I.V. Kravchenko, Burning and plasma chemistry, 5, 3 (2007)

10. V.V. Salomatov, I.V. Kravchenko, Burning and plasma chemistry, 5, 3 (2007)

11. V.V. Salomatov, Methods for calculating the nonlinear processes of heat transfer (Tomsk: Publishing house of Tomsk University Press, 1976)

12. D.A. Frank-Kamenetskiy, Diffusion and Heat Transfer in Chemical Kinetics (M.: Science, 1987)

13. V.N. Vilyunov, The theory of condensed matter ignition (Nauka Novosibirsk, 1984)

14. O.M. Todes, Zh.FH, 13, 7 (1939)

15. I.Z. Shtokalo, Operational calculus of generalization and applications of Kiev (Naukovadumka, 1972)

16. A.V. Lykov, The theory of thermal conductivity of the manual (M.: Higher School, 1967) 